\title{
Recurrence Rate of Carcinoma Breast patients Undergoing Mastectomy at Gulab Devi Hospital Lahore
}

\author{
MUHAMMAD ADIL IFTIKHAR ${ }^{1}$, KHALIL AHMED ${ }^{2}$, USMAN ALI RAHMAN ${ }^{3}$, MALIHAJAVAID BUTT $^{4}$, IFTIKHAR AHMED ${ }^{5}$, \\ MUHAMMAD KALEEM AKHTAR 6 \\ ${ }^{1}$ Assistant Professor Pediatric Surgery, Services Hospital Lahore \\ ${ }^{2}$ Professor of Surgery, Al-Aleem Medical College/Gulab Devi Hospital Lahore \\ ${ }^{3}$ Associate Professor Surgery, Al-Aleem Medical College/Gulab Devi Hospital Lahore \\ ${ }^{4}$ Senior Registrar, Surgical Unit 4, Services Hospital Lahore \\ ${ }^{5}$ Assistant Professor Surgery, Al-Aleem Medical College/Gulab Devi Hospital Lahore \\ ${ }^{6}$ Operation Theater Technologist, Gulab Devi Hospital Lahore \\ Correspondence to Dr. Muhammad Adil Iftikhar, Email muhammadayyanadil@gmail.com, Contact 0092-3338166714
}

\begin{abstract}
Aim: To determine recurrence rate of carcinoma breast after mastectomy in our population and to determine various risk factors associated with local recurrence which would help in management of new carcinoma breast patients in future.

Methods: It was a descriptive study conducted on patients of carcinoma breast with stage II and III undergoing modified radical mastectomy with negative resection margins at Gulab Devi Hospital, Lahore. Total of 59 patients were recruited in study using purposive sampling technique.

Results: Mean age was 51.61 11.52 years with range from 26 to 78 year. Female 58(98.31\%) predominated over the $1(1.69 \%)$ male. Recurrence rate was seen only in $5(8.47 \%)$ female patients

Conclusion: Proper surgical technique results in less chances of carcinoma breast recurrence. Menopause, positive axillary lymphnodes, lymphovascular invasion and necrosis on histology are associated factors of recurrence.

MeSH: Carcinoma breast, recurrence, Nottingham Prognostic Index (NPI)
\end{abstract}

\section{INTRODUCTION}

Carcinoma breast is the commonest cancer among female. According to American Cancer Society breast cancer was $30 \%$ of all newly diagnosed cancers. It also accounted for $15 \%$ of all estimated cancer deaths ${ }^{1}$. There are different pre disposing factors of breast cancer which include obesity, positive family history $(3.2 \%)$, previous breast pathology $(0.3 \%)$, age of menarche, lactation and age of mother at first child².

The breast is composed of mammary glands which are surrounded by a stroma of connective tissues. Each mammary gland is a type of modified sweat gland. It consists of number of ducts and secretory lobules. Every lobule is a formed from many alveoli which drain through single lactiferous duct. All lactiferous ducts converge at level of nipple similar to spokes of abicycle wheel. Breast consists of connective tissue stroma which has fibrous and fatty components. Other breast tissue stroma is fibrous which form ligaments. A layer of loose connective tissue lies between the breast and pectoral fascia. It is known as retro mammary space. This is a potential space used in breast conservation surgery. Metastasis of breast cancer occurs mostly through its lymphatics to regional lymph nodes. Each breast is drained by group of three lymph nodes which areaxillary nodes, posterior intercostal nodes and parasternal nodes. Major lymphatic drainage is through axillary lymph nodes ${ }^{3}$.

On histopathology; if breast carcinoma arises from lactiferous ducts is labelled as ductal carcinoma whereas carcinoma arising from lobules is called as lobular

Received on 07-02-2021

Accepted on 29-05-2021 carcinoma. Other two variants are mucinous and papillary carcinoma of breast. ${ }^{4}$ Ductal carcinoma in situ (DCIS) and invasive ductal carcinoma (IDC) are two most common types of ductal carcinoma. Management of carcinoma breast depends upon stage of cancer. Stage 1 and 2 breast cancers are usually managed with breast conserving surgery with adjuvant radiotherapy. In stage 3 breast carcinoma mastectomy, adjuvant chemotherapy and radiotherapy are opted as management. If breast conservative surgery is required neo-adjuvant chemotherapy and radiotherapy are given to down stage the tumor before undergoing surgery. In stage 4 metastatic simple mastectomy is performed. ${ }^{5}$

After mastectomy, many complications occurs, it includes risk of local recurrence, injury or thrombosis of axillary vein, seroma formation, shoulder dysfunction, pain \& numbness, flap necrosis, infection, lymphedema, axillary hyperesthesia and Winged Scapula ${ }^{6}$.

Main causes of recurrence after mastectomy include lymph node involvement. Larger tumors size has a greater risk of recurrence ${ }^{7}$. Lack of chemotherapy and radiation therapy has an increased risk of local breast cancer recurrence. Patient with inflammatory carcinoma have higher risk of local recurrence of carcinoma breast. Younger patients whose age is below 35 years at the time of initial breast cancer diagnosis, faces a high risk of recurrence for breast cancer. Positive resection margins in excised sample of cancer tissue can also raise the risk for recurrence.

The average recurrence rate after carcinoma breast surgery is $7-11 \%$. Local recurrence rate is high in case of breast conservative surgery as compared to mastectomy. The survival rate for patients is same in case of breast conservative surgery as compared to radical mastectomy ${ }^{8}$. 
The Nottingham prognostic index (NPI) is mainly used for prognosis of post mastecomy patients. It is calculated from tumor size, amount of positive lymph nodes and histological grading of the tumor. The five year survival rate of NPI I, II, III and IV are 93\%, 85\%, 70\%, 50\% respectively. ${ }^{9}$

\section{METHODS}

It was a descriptive study conducted at surgical department Gulab Devi Hospital Lahore. Non probability purposive sampling technique was used. The study was approved by our Ethical Committee. Sixty patients with carcinoma breast stage 2 \& 3 undergoing modified radical mastectomy were included in the study and were followed up for 02 years for any recurrence and to determine 02 year survival rate. Patients who developed lump or swelling at incision site after mastectomy for carcinoma breast and histologically diagnosed as malignancy were taken as recurrent carcinoma breast. The collected data was entered and analyzed by using SPSS version 25 . The qualitative data like gender was presented in the form of pie chart and tables with its percentage and quantitative data like age, recurrence rate and survival rate was presented in the form of frequency distribution mean, range and standard deviation.

\section{RESULTS}

A number of 59 participants were recruited through nonprobability sampling method. An adopted close ended

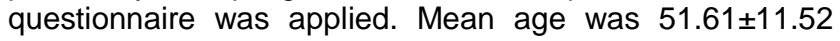
years with range from 26 to 78 year. It was also found that all of the $59(100 \%)$ participants were married. Female $58(98.31 \%)$ predominated over the $1(1.69 \%)$ male. It was also found that $21(36.2 \%)(n=58)$ participants were having menstrual cycle and $37(63.8 \%)(n=58)$ participants were menopause. Common comorbidities include hypertension 11(18.64\%), Diabetes mellitus 5(8.47\%), 6(10.17\%) had both hypertension and diabetes mellitus, Hepatitis $\mathrm{C}$ in $5(8.47 \%)$ participants. It was also found that only $3(5.08 \%)$ participants had family history of breast carcinoma. Results findings also show that $17(28.8 \%)$ participants were stage IIA, 10(16.95\%) participants were stage IIB, 16(27.12\%) participants were stage IIIA, $4(6.78 \%)$ participants were stage IIIB, 12(20.3\%) participants were stage IIIC (Fig.1).

Neoadjuvant chemotherapy was given in $41(69.49 \%)$ patients. The mean number of cycles for Neoadjuvant chemotherapy was 4.42. Adjuvant chemotherapy was given to $8(13.56 \%)$ patients. Neoadjuvant radiotherapy was given to $13(22.03 \%)$ patients. Adjuvant radiotherapy was given to 13(22.03) patients. Out of 59 participants, all 59 participants (100\%) had resection margins cleared.

Axillary lymph nodes were positive for malignancy in $43(72.8 \%)$ patients with the mean of axillary lymph node positive 4.69 \pm 5.7 . Results also revealed that, 56(94.92\%) patients had invasive ductal carcinoma, $2(3.39 \%)$ had invasive lobular carcinoma and $1(1.69 \%)$ had mucinous carcinoma type histopathology. Result findings also show that $47(79.66 \%)$ patients showed lymphovascular invasion while $5(8.47 \%)$ had tissue necrosis.

It was also found that $5(8.47 \%)$ patients had recurrence. All recurrence was seen among female patients. All female patients were menopause. All had mastectomy of right breast. Positive axillary lymph nodes for malignancy were seen in 3 patients having recurrence. Lymphovascular invasion and necrosis on histology were seen in all 5 patients having recurrence of carcinoma breast.

Figure 1 Frequency of stage of carcinoma breast patients

$\square$ Stage IIA $\square$ Stage IIB $\square$ Satge IIIA $\square$ Satge IIIB $\square$ Satge IIIC

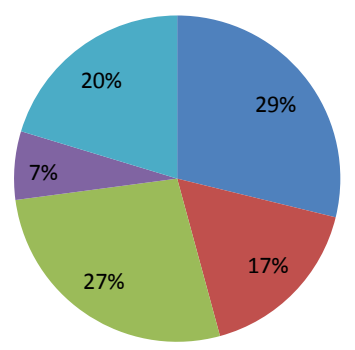

Figure 2. Recurrence rate of carcinoma breast patients

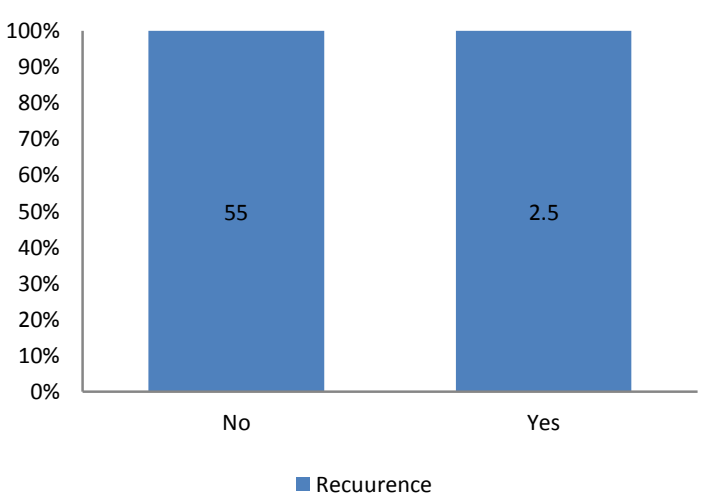

\section{DISCUSSION}

Breast carcinoma is the most common cancer among females. Number of positive axillary lymph nodes for tumor and size of primary tumor are accepted factors related for local recurrence. ${ }^{10} \mathrm{~A}$ randomized trial by Danish Breast Cancer Cooperative Group showed that larger size of primary tumor, histological ductal carcinoma, increase grade, fascial invasion, less amount of nodes removal during surgery, larger number of positive malignant lymph nodes, and extra-capsular invasion are all associated to increased risk of having local recurrence of carcinoma breast after mastectomy in auni-variate analyse ${ }^{11}$. As in our research project, predisposing factors for recurrence are menopausal women, positive axillary lymph nodes, presence of lympho-vascular invasion and higher grade indicated by tumor necrosis on histology.

In a retrospective research project of Jagsi et al, ${ }^{12}$ more than 870 cases of tumor negative lymph node with carcinoma breast had mastectomy, with nopostoperativeradiation. According to them tumor size 
more than $2 \mathrm{~cm}$, margins less than $2 \mathrm{~mm}$, pre-menopausal woman and lympho-vascular invasion are important indicators in determining prognosis of local breast recurrence. Ten-year local recurrence rate in patients with no risk factors was only $1.2 \%$ while in patients having only one risk factor the local recurrence rate was $10 \%$.Similarly patients having two risk factors had $17.9 \%$ and $40.6 \%$ patients had local recurrence having three risk factors. In $80 \%$ patients having recurrence was seen in chest wall. ${ }^{12}$ Youngerage group is also a major risk factor in predicting local recurrence of carcinoma breast. ${ }^{13}$

Recurrence rate in our study is $8.47 \%$ which is same as documented (7-11\%) in international and national studies. Recurrence was seen only in female patients as they were predominant. There was no significant effect of adjuvant and neoadjuvant radiotherapy and chemotherapy on recurrence of disease in our study. In our research project predisposing factors for recurrence; menopausal women, positive axillary lymph nodes, presence of lymphovascular invasion and higher grade indicated by tumor necrosis on histology were similar to factors documented in both national and international studies.

\section{CONCLUSION}

Risk factors associated with local recurrence of breast cancer play very important part in the governing of management of carcinoma breast. This governing can be rationalized if patients having more risk of developing local tumor recurrence can be diagnosed preoperatively. Currently, most commonly used methods are clinical parameters, histopathologic findings and many immunehistochemical markers. Carcinoma breast recurrence can also be prevented with proper surgical procedures ending in all negative resection margins. Special efforts should be made in menopausal patients and palpable axillary lymph nodes as they both are important predisposing factors in occurrence of recurrence in carcinoma breast patients.

\section{Conflict of interest: Nil}

\section{REFERENCES}

1. R. Siegel, K. Miller, and A. Jemal, "Cancer statistics 2019," Ca-Cancer J. Clin. 69(1), 7-34 (2019).
2. Martínez GA, Cano PR, González SH, Rosales FH. Predisposing factors to breast cancer in the Region Langunera (Mexico). Ginecologia y obstetricia de Mexico. 2004;72:525-30.

3. Fregnani JH, Macéa JR. Lymphatic Drainage of the Breast: from Theory to Surgical Practice. International journal of morphology. 2009 Sep 1;27(3).

4. Motlagh $\mathrm{MH}$, Jannesari $\mathrm{M}$, Aboulkheyr $\mathrm{H}$, Khosravi $\mathrm{P}$, Elemento O, Totonchi M, Hajirasouliha I. Breast cancer histopathological image classification: A deep learning approach. BioRxiv. 2018 Jan 1:242818.

5. Anjum F, Razvi N, Masood MA. Breast cancer therapy: a mini review. MOJ Drug Des Develop Ther. 2017;1(2):00006.

6. Luvisa K, Fan KL, Black CK, Wirth P, Won Lee D, Del Corral G, Willey SC, Song DH. Does surgeon handedness or experience predict immediate complications after mastectomy? A critical examination of outcomes in a single health system. The breast journal. 2020 Mar;26(3):376-83.

7. Spronk I, Schellevis FG, Burgers JS, de Bock GH, Korevaar JC. Incidence of isolated local breast cancer recurrence and contralateral breast cancer: a systematic review. The Breast. 2018 Jun 1;39:70-9.

8. Kheradmand AA, Ranjbarnovin N, Khazaeipour Z. Postmastectomylocoregional recurrence and recurrence-free survival in breast cancer patients. World journal of surgical oncology. 2010 Dec;8(1):1-7.

9. Zaimi A, Brahmi SA, Afqir S. Nottingham Prognostic Index is an Applicable Prognostic Tool in Non-Metastatic TripleNegative Breast Cancer. Asian Pacific journal of cancer prevention: APJCP. 2019;20(1):59.

10. Clarke $M$, Collins $R$, Darby $S$, et al: Effects of radiotherapy and of differences in the extent of surgery for early breast cancer on local recurrence and 15-year survival: An overview of the randomised trials. Lancet 366:2087-2106, 2005.

11. Nielsen HM, Overgaard M, Grau C, et al: Loco-regional recurrence after mastectomy in high-risk breast cancer-Risk and prognosis. An analysis of patients from the DBCG 82 b\&c randomization trials. Radiotherm Oncol 79:147-155, 2006.

12. Jagsi R, Raad RA, Goldberg S, et al: Locoregional recurrence rates and prognostic factors for failure in node-negative patients treated with mastectomy: Implications for postmastectomy radiation. Int $\mathrm{J}$ RadiatOncolBiolPhys 62:1035-1039, 2005.

13. de Bock GH, van der Hage JA, Putter $\mathrm{H}$, et al: Isolated locoregional recurrence of breast cancer is more common in young patients and following breast conserving therapy: Long-term results of European organisation for research and treatment of cancer studies. Eur J Cancer 42:351-356, 2006. 